\title{
Selection of Suitable Maximum-heart-rate Formulas for Use with Karvonen Formula to Calculate Exercise Intensity
}

\author{
Jinhua She $^{1} \quad$ Hitoshi Nakamura ${ }^{1} \quad$ Koji Makino $^{2} \quad$ Yasuhiro Ohyama $^{1} \quad$ Hiroshi Hashimoto $^{3}$ \\ ${ }^{1}$ Graduate School of Bionics, Computer and Media Sciences, Tokyo University of Technology, 1404-1 Katakura, \\ Hachioji, Tokyo 192-0982, Japan \\ ${ }^{2}$ Interdisciplinary Graduate School of Medical and Engineering, University of Yamanashi, 4-3-11, Takeda, Kofu 400-8511, Japan \\ ${ }^{3}$ Master Program of Innovation for Design and Engineering, Advanced Institute of Industrial Technology, 1-10-40 Higashiooi, \\ Shinagawa-ku, Tokyo 140-0011, Japan
}

\begin{abstract}
The Karvonen formula, which is widely used to estimate exercise intensity, contains maximum heart rate, $H R_{\max }$, as a variable. This study employed pedaling experiments to assess which of the proposed formulas for calculating $H R_{\max }$ was the most suitable for use with the Karvonen formula. First, two kinds of experiments involving an ergometer were performed: an all-in-one-day experiment that tested eight pedaling loads in one day, and a one-load-per-day experiment that tested one load per day for eight days. A comparison of the data on 7 subjects showed that the all-in-one-day type of experiment was better for assessing $H R_{\max }$ formulas, at least for the load levels tested in our experiments. A statistical analysis of the experimental data on 47 subjects showed two of the $H R_{\max }$ formulas to be suitable for use in the Karvonen formula to estimate exercise intensity for males in their $20 \mathrm{~s}$. In addition, the physical characteristics of a person having the greatest impact on exercise intensity were determined.
\end{abstract}

Keywords: Borg CR10 scale, Karvonen formula, exercise intensity, maximum heart rate, pedaling, statistical analysis.

\section{Introduction}

Two common measures of exercise intensity are oxygen intake and heart rate ${ }^{[1,2]}$. Oxygen intake is the amount of oxygen that the body takes in during respiration; and oxygen intake per kilogram of body weight per minute, $\dot{\mathrm{V}} \mathrm{O}_{2}$, can be used to calculate exercise intensity ${ }^{[3]}$. On the other hand, heart rate, $H R$, is used in the Karvonen formula to calculate exercise intensity ${ }^{[3]}$. While the measurement of oxygen intake needs expertise and a large apparatus, heart rate is easy to measure with a small instrument and even in a remote fashion ${ }^{[4]}$. So, the Karvonen formula is widely used in the fields of rehabilitation and physical training.

One of the variables in the Karvonen formula is maximum heart rate, $H R_{\max }$, which is the heart rate a person has when he pushes his body to the limit. Since directly measuring $H R_{\max }$ not only takes a great deal of time, but also imposes a heavy physical burden on the subject, a simple, convenient formula based on a person's age ${ }^{[5]}$ is extensively used nowadays ${ }^{[6-8]}$ to calculate it:

$$
H R_{\max }=220-\text { age }
$$

where "age" is the age of the subject. However, Robert and Landwehr ${ }^{[5]}$ pointed out that (1) does not always yield the correct $H R_{\max }$. Although several methods have been

Regular paper

Manuscript received October 24, 2013; accepted January 14, 2014

This work was supported by Health Science Center Foundation, Japan.

Recommended by Associate Editor Min Wu

(C)Institute of Automation, Chinese Academy of Science and Springer-Verlag Berlin Heidelberg 2015 proposed to improve the accuracy, none of them is widely recognized; and their range and conditions of use are not clear.

The aim of this study was to select the methods of calculating the $H R_{\max }$ of a person pedaling a cycle ergometer that are suitable for use with the Karvonen formula. We measured a person's heart rate while he was pedaling under various loads, and obtained his rating of perceived exertion (RPE) before and after each pedaling experiment from a questionnaire. Then, based on a comparison of the data from the experiments and questionnaires, we chose the most appropriate methods of calculating $H R_{\max }$. To ensure accuracy and to determine how the work load immediately prior to exercise influences exercise intensity, we performed two kinds of pedaling experiments: an all-in-oneday (AIOD) experiment that tested all pedaling loads in one day, and a one-load-per-day (OLPD) experiment that tested one load per day for several days. Then, we examined the differences in exercise intensity between these two kinds of experiments, and assessed whether or not the OLPD experiment was needed. Finally, based on the experimental and questionnaire data, we made clear the degree of influence of some of a person's physical characteristics on exercise intensity.

In this study, the advisability of the experiments was first assessed by the ethics committee of the Tokyo University of Technology. Prospective subjects for the experiments were given an oral explanation and descriptive printed material on how the data and personal information acquired during the experiments would be handled, and their consent 
was obtained before their participation in the experiments was allowed. All the subjects were students, who were covered by personal accident insurance for students pursuing education and research, which was provided by Japan Educational Exchanges and Services. This paper is a considerable improvement over our previous one ${ }^{[9]}$. We substantially refined and expanded the work by collecting experimental data and performing a thorough investigation of the selection of physical characteristics that are related to exercise intensity.

\section{Exercise intensity and $H R_{\max }$}

Exercise intensity indicates the degree of difficulty of exercise. There are two kinds of exercise intensity: Physiological exercise intensity and RPE.

Even though RPE is a subjective measure, it is in good agreement with the physiological exercise intensity ${ }^{[10]}$; and it is commonly used to obtain a subject's impression of the degree of difficulty. The Borg CR10 scale ${ }^{[11]}$ (Fig. 1) is used as a measure of RPE. It has the advantage that its relationship to exercise intensity is intuitively understandable. It has values in the range $[0,10]$, with larger values indicating greater intensity.

\begin{tabular}{|c|c|c|c|c|c|c|c|c|c|}
\hline $\begin{array}{r}\text { Seating o } \\
\text { standing } \\
\text { calmly }\end{array}$ & $\begin{array}{l}\text { I } \\
\text { Walking }\end{array}$ & Ligh & $\begin{array}{l}\text { louse } \\
\mathrm{k}\end{array}$ & $\begin{array}{r}\text { Maint } \\
\text { ph }\end{array}$ & ing/ & $\begin{array}{l}\text { aproving } \\
\text { ength }\end{array}$ & & $\begin{array}{r}\text { Reinf } \\
\text { tran } \\
\text { of ath }\end{array}$ & \\
\hline $\begin{array}{llll}0 & 0.5 & 1\end{array}$ & 2 & 3 & 4 & 5 & 6 & 7 & 8 & 9 & 10 \\
\hline 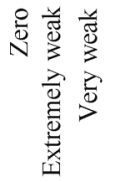 & $\frac{\mu}{\sqrt[0]{0}}$ & $\frac{0}{\frac{\pi}{0}}$ & & $\begin{array}{l}\stackrel{00}{0} \\
\stackrel{5}{5}\end{array}$ & & 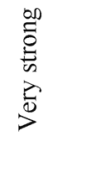 & & & 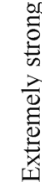 \\
\hline
\end{tabular}

Fig. 1 Borg CR10 scale ${ }^{[11]}$

The physiological exercise intensity is based on a physiological index related to oxygen intake or heart rate ${ }^{[12]}$.

Let the maximum oxygen intake be $\dot{V} O_{2 \max }$. Then, the relative oxygen intake is

$$
\dot{V} O_{2} \%=\frac{\dot{V} O_{2}}{\dot{V} O_{2} \max } \times 100 \%
$$

$\dot{V} O_{2}$ increases as exercise intensity increases, making it suitable for the evaluation of physiological exercise intensity. Since this method needs a large apparatus and takes a long time, it is mainly used in well-equipped laboratories and is difficult to use outdoors. Furthermore, the measurement of $\dot{V} O_{2}$ max pushes the body to the limit, which can entail some danger to the subject.

In contrast, it is much easier and cheaper to measure $H R$ than $\dot{V} O_{2}$. This is why the heart-rate-based Karvonen formula is widely used in rehabilitation, physical training, and other fields. The relationship between $\dot{V} O_{2}$ and $H R$ is

$$
\dot{V} O_{2}=H R \times A V D O_{2} \times S V
$$

where $A V D O_{2}$ is the arteriovenous oxygen difference; and $S V$ is stroke volume, which is the volume of blood pumped out of one ventricle of the heart with each beat. It is known that $A V D O_{2}$ and $S V$ converge to particular values regardless of the exercise load, and $H R$ increases as exercise intensity increases. Since $\dot{V} O_{2}$ is proportional to $H R$, we can use $H R$ instead of it to calculate exercise intensity ${ }^{[13]}$.

This study is focused on the Karvonen formula,

$$
H R R \%=\frac{H R-H R_{r}}{H R_{\max }-H R_{r}} \times 100 \%
$$

where $H R_{r}$ is the subject's heart rate at rest and $\% H R R$ is the heart rate reserve (HRR).

We first measure a subject's heart rate, $H R$. Then, we calculate the $H R R$ for the RPE. $H R R$ is in the range $[0,100]$, and it is proportional to a value on the Borg CR10 scale, $\left.B_{10}{ }^{[11} 14\right]$ :

$$
H R R=10 \times B_{10} .
$$

Remark 1. The calculation of $\% H R R$ in (4) uses only two parameters: maximum heart rate and the heart rate at rest; that is, it does not take into consideration a subject's height, weight, body mass index $(\mathrm{BMI})^{1}$, or other physical characteristics. Thus, the calculated value of $\% H R R$ may be less accurate for an older person than for a younger person $^{[15]}$. The final goal of our research is to improve the Karvonen formula by incorporating information on physical characteristics so as to adapt $\% H R R$ individually to each subject.

$H R_{\max }$ in the Karvonen formula is often calculated using (1). However, questions have arisen concerning the accuracy of the $H R_{\max }$ given by (1). Robert and Landwehr ${ }^{[5]}$ verified the original data used to obtain (1) and pointed out that it was possible that (1) might not give the correct $H R_{\max }$. A large number of studies have attempted to improve (1). Inbar et al. ${ }^{[16]}$, for example, had 1424 healthy subjects perform treadmill exercises. They clarified that $H R_{\text {max }}$ decreases by $0.685 \mathrm{bpm}$ per year due to aging, and proposed the formula

$$
H R_{\text {max }}=205.8-0.685 \times \text { age } .
$$

Miller et al. ${ }^{[17]}$ showed the equation

$$
H R_{\max }=217-0.85 \times \text { age }
$$

based on exercise by 86 obese and 51 normal-weight adults. Tanaka et al. ${ }^{[18]}$ pointed out the problem that insufficient data on the elderly was used to derive maximum-heart-rate formulas. They examined 351 samples involving 492 groups and 18712 subjects, and came up with

$$
H R_{\max }=208-0.7 \times \text { age. }
$$

Gulati et al. ${ }^{[19]}$ speculated that $H R_{\max }$ should be different for men and women. They carried out exercise tests on 5437 asymptomatic women and came up with

$$
H R_{\max }=206-0.88 \times \text { age } .
$$

\footnotetext{
${ }^{1} B M I=\frac{W}{H^{2}}$ where $W(\mathrm{~kg})$ is body weight and $H(\mathrm{~m})$ is height.
} 
Londeree and Moeschberge ${ }^{[20]}$ pointed out that (4) does not account for a person's physical characteristics (weight, height, etc.) and thus may not yield the correct $H R_{\max }$. Taking age, sex, load level, and other factors into consideration, they suggested

$$
H R_{\max }=206.3-0.711 \times \text { age }
$$

based on data collected from world-class athletes.

So, there are many methods of calculating $H R_{\max }$; and we need to determine which of them are suitable for use in the Karvonen formula. Furthermore, since (4) does not contain any personal information except heart rate, the calculated exercise intensity may not be accurate for each person $^{[15]}$.

\section{$3 \quad$ Pedaling exercise and analysis}

This study employed a pedaling exercise on a cycle ergometer to achieve two goals:

1) to compare (1) and (6)-(10), and find the ones most suitable for calculating exercise intensity for a pedaling exercise; and

2) to show the degree of relationship between exercise intensity and physical characteristics by dividing the relationships into two groups based on linear and nonlinear correlations.

This section explains the pedaling exercises used in this study and presents an analysis of the data obtained.

\subsection{Experiments}

We used a cycle ergometer (Programmable Ergometer AFB6008; Alinco, Inc.) for pedaling experiments and a photoelectric pulsometer (Pulse Coach Neo HR-40; Japan Precision Instruments, Inc.) to record a person's pulse rate during an experiment (Fig. 2). The ergometer calculates the work load and displays the result on a panel. Note that pulse rate is the same as heart rate for healthy people. All the experiments were carried out in our laboratory.

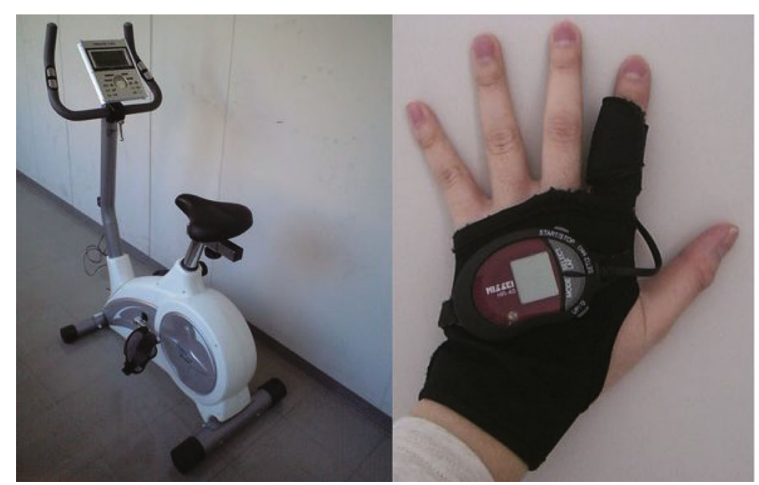

Fig. 2 Left: programmable ergometer, AFB6008; Right: photoelectric heart rate meter, Pulse Coach Neo HR-40

The ergometer can be set to any of 16 pedaling loads (1)-(16) by pushing up or down buttons. It is necessary to identify the relationship between pedaling load and actual work load so that readers can understand what a particular pedaling load means. Thus, prior to the pedaling experiments, we performed preliminary experiments on pedaling load in which two subjects (age: 22 years old; sex: male; health: good) pedaled the ergometer for 5 minutes at a speed of about $60 \mathrm{rpm}$. The experimental results (Fig. 3) show that the work load increases $15 \mathrm{~W}$ for every unit increase in pedaling load.

In the main pedaling experiments, due to fatigue and scheduling considerations, subjects were only tested at eight of the sixteen load levels: 1, 3, 5, 7, 9, 11, 13, and 15 .

Our daily experience tells us that fatigue influences exercise intensity. However, we could not find any reports related to this issue. So, we examined this issue through two kinds of experiments: AIOD and OLPD. An AIOD experiment tested all eight pedaling loads in one day, and an OLPD experiment tested one load per day for eight days. 47 subjects (university students; age: 20-29; sex: male; health: good) took part in the AIOD experiment, and 7 of them also took part in the OLPD experiment (Tables 1 and 2). Note that the average weight of most Japanese males is $66.75 \mathrm{~kg}$ for the age range $20-24$ and $66.57 \mathrm{~kg}$ for the range $25-29^{[21]}$. A $t$-test on the difference between the subjects' weights and the average value of the statistical data $^{[21]}$ shows that, for a $p$-value of 0.05 , there is no significant difference between them.

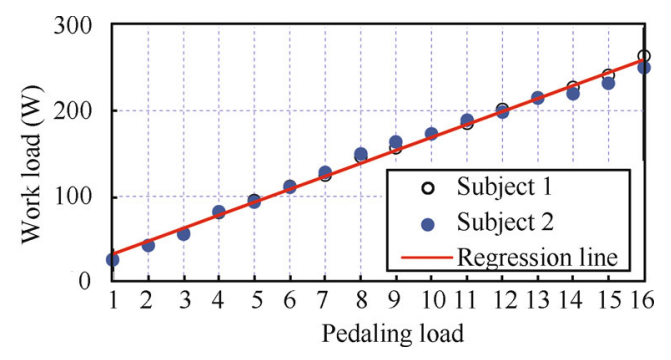

Fig. 3 Work load vs. pedaling load

Table 147 subjects for all-in-one-day (AIOD) experiment (SD: standard deviation)

\begin{tabular}{ccccc}
\hline & Min & Max & Average & SD \\
\hline Age (year) & 20 & 29 & 23.1 & 2.7 \\
Height (cm) & 158 & 185 & 172.3 & 6.2 \\
Weight (kg) & 41 & 92 & 66.3 & 12.4 \\
$H R_{r}$ (bpm) & 62 & 100 & 78.3 & 10.4 \\
Health & Good & Good & Good & - \\
\hline
\end{tabular}

Table 27 subjects for one-load-per-day (OLPD) experiment (SD: standard deviation)

\begin{tabular}{ccccc}
\hline & Min & Max & Average & SD \\
\hline Age (year) & 21 & 22 & 21.3 & 0.5 \\
Height $(\mathrm{cm})$ & 158 & 178 & 168.0 & 8.2 \\
Weight $(\mathrm{kg})$ & 49 & 76 & 61.6 & 11.2 \\
$H R_{r}(\mathrm{bpm})$ & 66 & 98 & 82.6 & 11.4 \\
Health & Good & Good & Good & - \\
\hline
\end{tabular}

The procedures for the two types of experiments are given below. 
AIOD experiment:

Step 1. Before the experiment, give the subject a questionnaire (Fig. 9 in Appendix) to collect data on physical characteristics.

Step 2. Mount the pulsometer on the subject's hand and have the subject take a one-min rest.

Step 3. Set the sampling time for the measurement of pulse rate to $4 \mathrm{~s}$.

Step 4. Measure the pulse rate at rest for 1 minute and repeat the measurement 5 times.

Step 5. Set the load of the ergometer to Level 1.

Step 6. Have the subject pedal the ergometer at a speed of about $60 \mathrm{rpm}$ for 5 minutes, and record the pulse rate (Fig. 4).

Step 7. After the experiment, use a questionnaire (Fig. 10 in Appendix) to collect data on perceived exercise intensity (PEI). Give the subject a 5-min rest and then record the pulse rate.

Step 8. Increase the load level by 2 and go to Step 6 . Repeat Steps 6-8 up to the maximum load or until the subject feels that he has reached the limit of his strength.

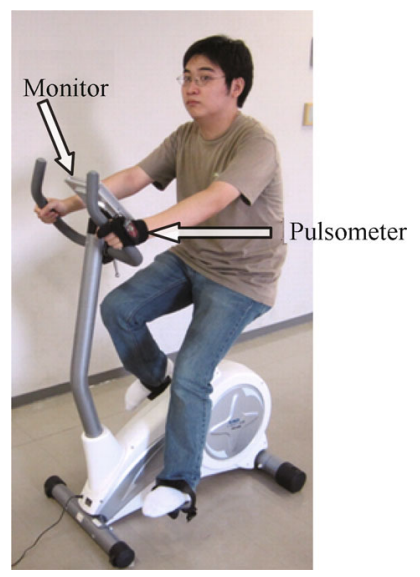

Fig. 4 Photograph of experiment in progress

The post-experiment questionnaire asks a subject to choose an appropriate level on the Borg CR10 scale. This is taken to be his RPE.

OLPD experiment:

Step 1. Give the subject a questionnaire before the experiment (Fig. 9 in Appendix).

Step 2. Set the load of the ergometer to Level 1 on the first day and increase the load level by 2 on each succeeding day (2nd day: Level 3; 3rd day: Level 5; etc.).

Step 3. Have the subject pedal the ergometer at a speed of $60 \mathrm{rpm}$ for 5 minutes and record the pulse rate.

Step 4. Give the subject a questionnaire (Fig. 10 in Appendix) after the experiment to obtain the PEI. This is the end of the experiment for that day.

Step 5. Repeat Steps 1-4 for 8 days or until the subject feels that he has reached the limit of his strength.

We call the exercise intensity calculated from the Karvonen formula plus the experimental data the calculated exercise intensity (CEI), and we call the value obtained from the questionnaire the PEI. We performed a least-squares analysis of the CEI and PEI and examined the relationship between exercise intensity and work load. Two parameters are used to describe the relationship between CEI (or PEI) and work load (Fig. 5): the slope, $a_{c}$ (or $a_{p}$ ), and the ordinate intercept, $b_{c}\left(\right.$ or $\left.b_{p}\right)$.

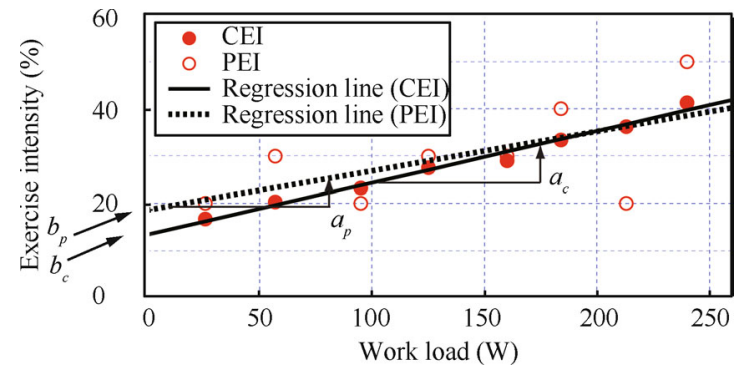

Fig. 5 Experimental results and regression lines of CEI and PEI

\subsection{Analysis of experimental data}

First, we compared the AIOD and OLPD results to determine the effect of immediately preceding work load on present exercise intensity. We identified the parameters $a_{c}$ and $b_{c}$, and $a_{p}$ and $b_{p}$ for both AIOD and OLPD using the $H R_{\max }$ calculated from (1) to determine if the settings in the experiments were suitable or not.

Since the subjects involved in the experiments were healthy (they neither underwent treatment at a hospital nor took medication), and since the pedaling loads in the experiments were so light that they did not interfere with daily life, significant fatigue did not accumulate in the body. Thus, we only needed to consider the recovery time for very short-term fatigue.

The 4 parameters $\left(a_{c}, b_{c}, a_{p}, b_{p}\right)$ were calculated for each subject for the AIOD and OLPD experiments. A $t$ test on the differences between the parameters for AIOD and OLPD showed that, for a $p$-value of 0.05 , there was no significant difference in exercise intensity between the two types of experiments. And a comparison of the parameters for AIOD and OLPD (Table 3) revealed the differences to be very small. Thus, we can conclude that the effect of immediately preceding work load on present exercise intensity is very small for our pedaling experiments in the range of work loads we used, and that the OLPD experiment is unnecessary for this study.

Next, we used the AIOD experiment to select appropriate methods of calculating $H R_{\text {max }}$ based on data collected from 47 subjects. Two criteria for the selection were examined: $\left(a_{c}-a_{p}\right)$ and $\left(b_{c}-b_{p}\right)$. A variance analysis of these two variables for (1) and (6)-(10) showed that they resulted in the selection of the same methods. So, we used only $\left(a_{c}-a_{p}\right)$ and carried out the selection as follows:

Procedure for selecting methods of calculating $H R_{\max }$ :

Step 1. Calculate $H R_{\max }$ using (1) for Subject 1, and obtain $a_{c}^{(1)}(1)$.

Step 2. Calculate the slope of PEI, $a_{p}(1)$, for Subject 1 .

Step 3. Calculate $\Delta a^{(1)}(1):=\left[a_{c}^{(1)}(1)-a_{p}(1)\right]^{2}$. 
Table 3 Mean and standard deviation of parameters of CEI and PEI for AIOD and OLPD

\begin{tabular}{ccccccccccccccc}
\hline & \multicolumn{3}{c}{$a_{c}$} & \multicolumn{1}{c}{$b_{c}$} & \multicolumn{4}{c}{$a_{p}$} & \multicolumn{4}{c}{$b_{p}$} \\
& AIOD & OLPD & Diff. & AIOD & OLPD & Diff. & AIOD & OLPD & Diff. & AIOD & OLPD & Diff. & & \\
\hline Average & 0.297 & 0.301 & -0.004 & 1.320 & 0.253 & 1.067 & 0.249 & 0.253 & -0.004 & 25.587 & 17.041 & 8.546 \\
SD & 0.077 & 0.094 & 0.068 & 5.066 & 0.079 & 5.129 & 0.098 & 0.079 & 0.118 & 10.457 & 7.499 & 12.201 & & \\
\hline
\end{tabular}

Step 4. Do Steps $1-3$ for all the subjects, and calculate $\Delta a^{(1)}(i):=\left[a_{c}^{(1)}(i)-a_{p}(i)\right]^{2}, i=2, \cdots, 47$.

Step 5. Calculate the mean value and the variance of $\Delta a^{(1)}(i), i=1, \cdots, 47$.

Step 6. Do Steps $1-5$ using (6)-(10) one by one.

Step 7. Analyze the results obtained in Steps 1-6, and assess the suitability of using the formulas to calculate $H R_{\max }$.

First, we examined the correlation between the BorgCR10-based PEI and the CEI obtained from (1) and (6)(10). Analysis of the data shows that all the coefficients for the correlation between PEI and CEI are larger than 0.995. This means that they are strongly correlated. Next, an $F$-test shows that, for a $p$-value of 0.05 , there is no significant difference for any $\Delta a^{(j)},(j=1,4,5, \cdots, 8)$, and that (1) and (7) give the smallest $\Delta a^{(j)}$ among the 6 methods. Thus, (1) and (7) are the most suitable methods of calculating exercise intensity for males in their $20 \mathrm{~s}$ (Table 4).

We also examined the relationship between exercise intensity and the following physical characteristics:

1) Height $(H)(\mathrm{m})$.

2) Weight $(W)(\mathrm{kg})$.

3) Hours of sleep $(H S)(\mathrm{h})$.

4) Heart rate at rest $\left(H R_{r}\right)(\mathrm{bpm})$.

5) BMI $\left(\mathrm{kg} / \mathrm{m}^{2}\right)$.

Table 4 Mean and standard deviation of $\Delta a^{(j)}(j=1,4,5, \cdots, 8)$ for various $H R_{\max }$ formulas (SD: standard deviation)

\begin{tabular}{lcc}
\hline$H R_{\max }$ formulas & Average & $\mathrm{SD}$ \\
\hline Equation (1): $H R_{\max }=220-$ age & $\mathbf{0 . 0 1 1}$ & $\mathbf{0 . 0 1 7}$ \\
Equation (6): $H R_{\max }=205.8-0.685 \times$ age & 0.014 & 0.022 \\
Equation (7): $H R_{\max }=217-0.85 \times$ age & $\mathbf{0 . 0 1 1}$ & $\mathbf{0 . 0 1 7}$ \\
Equation (8): $H R_{\max }=208-0.7 \times$ age & 0.013 & 0.020 \\
Equation (9): $H R_{\max }=206-0.88 \times$ age & 0.016 & 0.025 \\
Equation (10): $H R_{\max }=206.3-0.711 \times$ age & 0.014 & 0.022 \\
\hline
\end{tabular}

The parameters for excise intensity were:

1) Slope $\left(a_{p}\right)$ and ordinate intercept $\left(b_{p}\right)$ of PEI.

2) Slope $\left(a_{c}\right)$ and ordinate intercept $\left(b_{c}\right)$ of CEI.

3) The highest heart rate recorded during an experiment $\left(H R_{m}\right)$.

4) The difference between the highest heart rate and the heart rate at rest $\left(\Delta H R=H R_{m}-H R_{r}\right)$.

We calculated the correlation coefficients for 55 combinations of items in the two groups (Table 5). The number of subjects is $n=47$, and the number of degrees of freedom is $n-2=45$. So, when there is no correlation, $t_{0.05}=2.014$ for a $p$-value of 0.05 . This gives the critical value for correlation:

$$
\underline{r}=\frac{t_{0.05}}{\sqrt{n-2+t_{0.05}^{2}}}=0.288
$$

That is, there is a significant correlation between two items if the correlation coefficient is larger than $\underline{r}$. Sorting the items in descending order of correlation coefficient yields

1) $a_{c}: H R_{m}, b_{c}, \mathrm{BMI}, H R_{r}, a_{p}, \Delta H R$, and $W$.

2) $b_{c}: H R_{r}$ and $a_{p}$.

3) $a_{p}: b_{p}, \mathrm{BMI}$, and $W$.

4) $b_{p}:$ None.

Some relationships are illustrated in Figs. 6-8. Fig. 6 shows that $a_{c}$ decreases as BMI increases. Thus, a larger BMI means less sensitivity to an increase in work load. Fig. 7 also shows the same tendency in the relationship between $a_{p}$ and BMI. So, the larger a person's BMI is, the slower the increase in his heart rate is as the work load increases.

Fig. 8 shows that, when $H R_{m}$ is large, $a_{c}$ is large. Note that the correlation coefficient for these two items is larger than that for $a_{c}$ and BMI, and the amount of scatter is small. So, the CEI is more sensitive to an increase in work load if a person can endure a larger $H R_{m}$ during exercise.

Furthermore, we calculated the correlation ratios to examine the nonlinear relationships between the parameters $^{[22]}$. For each of the four parameters for excise

Table 5 Correlation coefficients between exercise intensity and parameters of physical characteristics $(p<0.05)$

\begin{tabular}{|c|c|c|c|c|c|c|c|c|c|c|}
\hline & $b_{c}^{(1)}$ & $a_{p}$ & $b_{p}$ & $H$ & $W$ & $H S$ & $H R_{r}$ & $H R_{m}$ & $\Delta H R$ & BMI \\
\hline$a_{c}^{(1)}$ & -0.558 & 0.437 & 0.031 & 0.015 & -0.426 & 0.052 & 0.455 & 0.725 & 0.435 & -0.504 \\
\hline$b_{c}^{(1)}$ & - & -0.319 & 0.170 & -0.212 & -0.024 & -0.278 & -0.384 & -0.190 & 0.056 & 0.069 \\
\hline$a_{p}$ & - & - & -0.583 & -0.052 & -0.346 & 0.212 & 0.237 & 0.117 & -0.035 & -0.375 \\
\hline$b_{p}$ & - & - & - & 0.064 & 0.049 & -0.117 & 0.111 & 0.186 & 0.115 & 0.027 \\
\hline$H$ & - & - & - & 一 & 0.506 & 0.128 & 0.129 & -0.036 & -0.119 & 0.149 \\
\hline$W$ & - & - & - & - & 一 & 0.192 & 0.021 & -0.216 & -0.230 & 0.926 \\
\hline$S H$ & - & - & - & - & 一 & - & 0.044 & 0.043 & 0.015 & 0.159 \\
\hline$H R_{r}$ & - & - & - & - & - & - & - & 0.324 & -0.316 & -0.045 \\
\hline$H R_{m}$ & - & - & - & - & - & - & - & - & 0.795 & -0.255 \\
\hline$\Delta H R$ & - & - & - & - & - & - & - & - & - & -0.227 \\
\hline
\end{tabular}


intensity $\left(a_{c}, b_{c}, a_{p}, b_{p}\right)$, we calculated its correlation ratio with ten other parameters for a total of 34 pairs (Table 6$)$. A correlation ratio in the range $[0.5,0.7]$ indicates a nonlinear relationship between two items. Sorting the items in descending order of correlation ratio yields:

1) $a_{c}: H R_{m}, a_{p}, b_{c}, \mathrm{BMI}$, and $W$.

2) $b_{c}: H R_{r}$.

3) $a_{p}: b_{p}, H R_{m}$, and BMI.

4) $b_{p}$ : None.

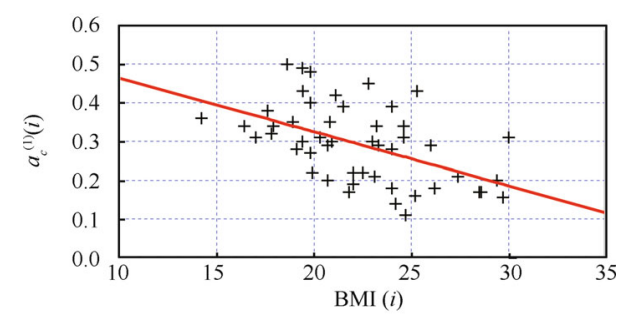

Fig. 6 Calculated slope vs. BMI ( $i$ : subject, $i \in\{1, \cdots, 47\})$

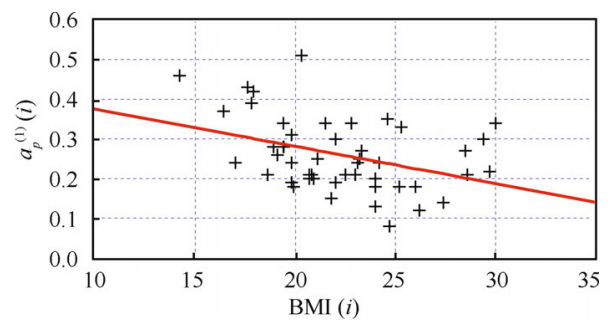

Fig. 7 Slope of RPE vs. BMI ( $i$ : subject, $i \in\{1, \cdots, 47\})$

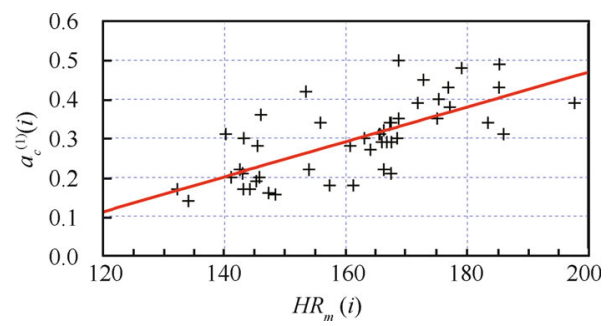

Fig. 8 Maximum heart rate at experimental vs. calculated slope (i: subject, $i \in\{1, \cdots, 47\}$ )

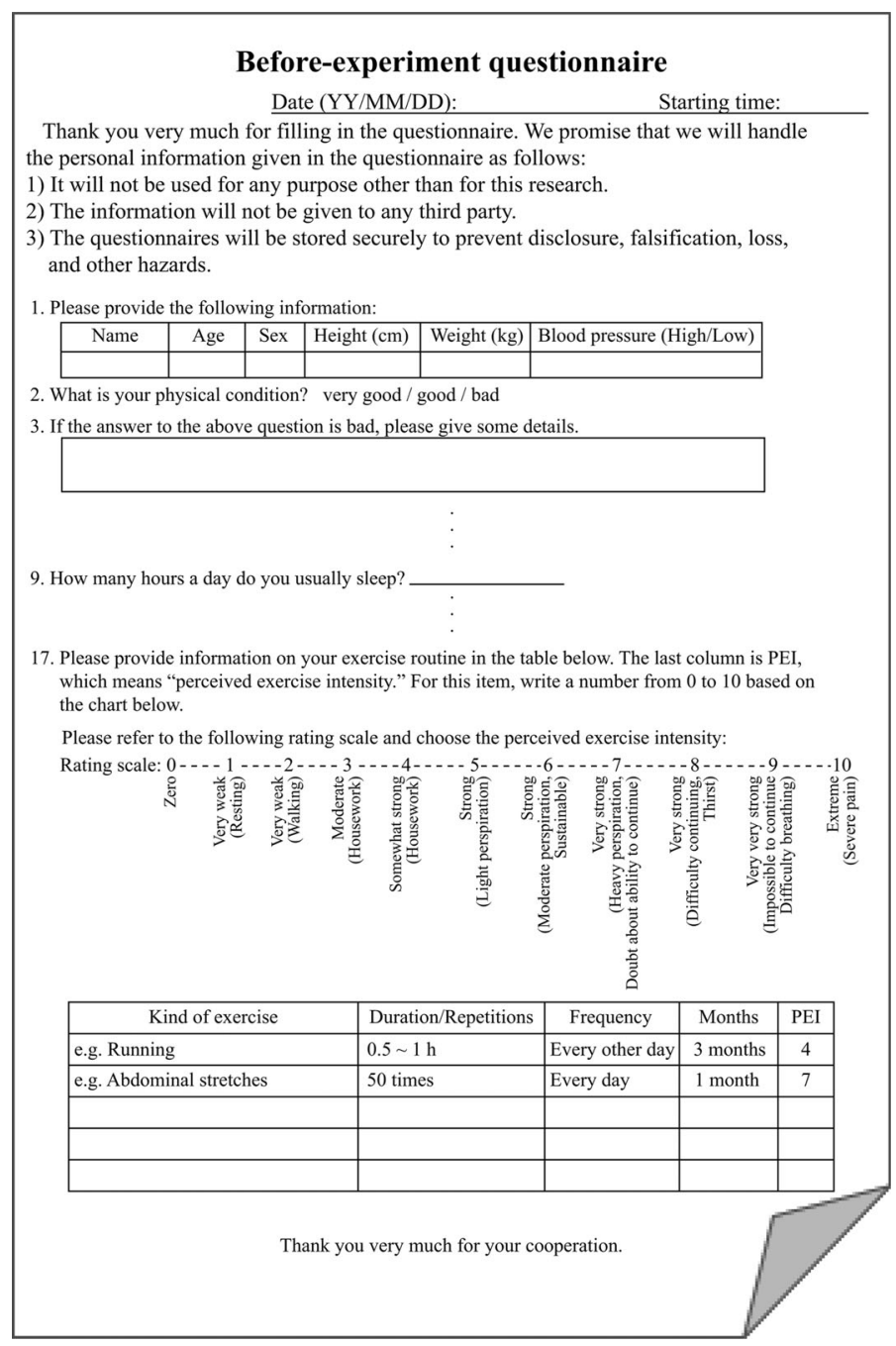

Fig. 9 Questionnaire before experiment 
Table 6 Correlation ratio between exercise intensity and the parameters of physical characteristics

\begin{tabular}{|c|c|c|c|c|c|c|c|c|c|c|}
\hline & $b_{c}^{(1)}$ & $a_{p}$ & $b_{p}$ & $H$ & $W$ & $H S$ & $H R_{r}$ & $H R_{m}$ & $\triangle H R$ & $B M I$ \\
\hline$a_{c}^{(1)}$ & 0.601 & 0.673 & 0.416 & 0.320 & 0.546 & 0.216 & 0.436 & 0.693 & 0.421 & 0.583 \\
\hline$b_{c}^{(1)}$ & - & 0.379 & 0.177 & 0.278 & 0.377 & 0.315 & 0.608 & 0.280 & 0.167 & 0.363 \\
\hline$a_{p}$ & - & - & 0.691 & 0.314 & 0.574 & 0.362 & 0.289 & 0.579 & 0.456 & 0.576 \\
\hline$b_{p}$ & - & - & - & 0.160 & 0.274 & 0.283 & 0.211 & 0.274 & 0.162 & 0.289 \\
\hline
\end{tabular}

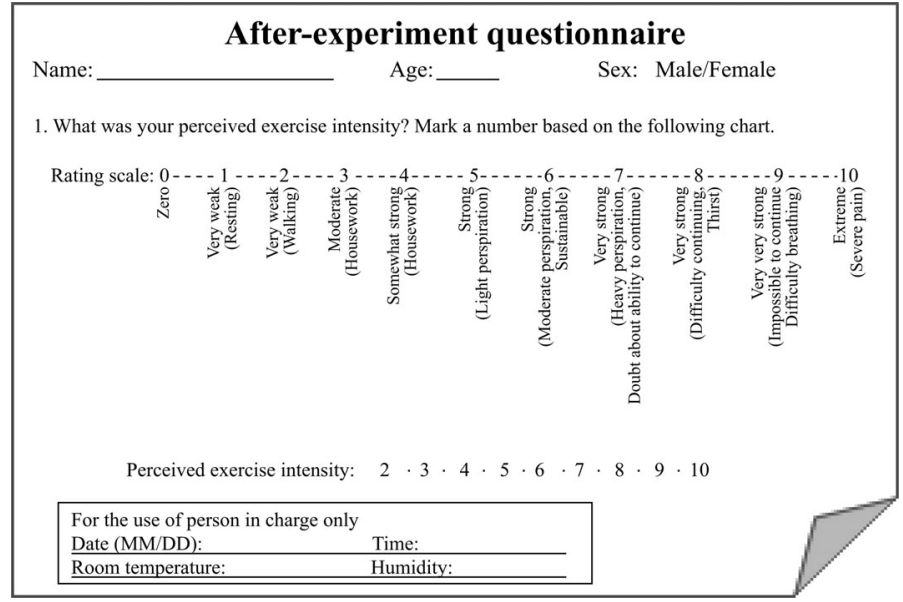

Fig. 10 Questionnaire after experiment

\section{Conclusions}

This study aimed to select the most suitable methods of calculating $H R_{\max }$ for use in the Karvonen formula, which provides an estimate of exercise intensity, and to examine the physical characteristics related to exercise intensity. We designed two kinds of pedaling experiments (AIOD, OLPD) and carried them out with male university students in their $20 \mathrm{~s}$. Based on the results of experiments and questionnaires for 47 subjects, we chose the difference between the slopes of CEI and PEI as the critical variable, and selected formulas for calculating $H R_{\max }$. We also analyzed the relationship between physical characteristics and the parameters for excise intensity. The following points were clarified:

1) For a $p$-value of 0.05 , there is no significant difference between the results of the AIOD and OLPD experiments. Thus, the OLPD experiment is unnecessary for the work loads used in these experiments; the AIOD experiment alone is sufficient.

2) Among the 6 methods of calculating $H R_{\max }$ that were tested, (1) and (7) were found to be the most suitable for male university students in their $20 \mathrm{~s}$.

3) The physical characteristics that are linearly and nonlinearly related to excise intensity were clarified through an analysis of correlation coefficients and correlation ratios. These results will help in choosing the factors suitable for adapting the CEI to individuals.

This paper reports results on the selection of suitable methods of calculating $H R_{\max }$ for use with the Karvonen formula as applied to pedaling exercises for people in their $20 \mathrm{~s}$. It is necessary to determine whether or not these methods are also suitable for other age groups. This will be examined and reported in the near future.
The final goal of our research is to modify the Karvonen formula by incorporating physical characteristics into it so as to adapt it to individuals. This is an interesting and challenging subject for future study.

\section{Appendix}

The main items in the before- and after-experiment questionnaires are shown in the following figures.

\section{References}

[1] A. M. Jones, D. C. Poole. Oxygen Uptake Kinetics in Sport, Exercise and Medicine, Oxon: Routledge, 2005.

[2] R. Benson, D. Connolly. Heart Rate Training, Champaign: Human Kinetics, 2011.

[3] D. S. Brooks. The Complete Book of Personal Training, Champaign: Human Kinetics, 2003.

[4] Y. Chen, P. Rapajic. Human respiration rate estimation using ultra-wideband distributed cognitive radar system. International Journal of Automation and Computing, vol. 5, no. 4, pp. 325-333, 2008.

[5] R. A. Robert, R. Landwehr. The surprising history of the "HRmax $=220-$ age" Equation. Journal of Exercise Physiologyonline, vol. 5, no. 2, pp. 1-10, 2002.

[6] S. Young-McCaughan, S. M. Arzola. Exercise intervention research for patients with cancer on treatment. Seminars in Oncology Nursing, vol. 23, no. 4, pp. 264-274, 2007.

[7] S. Shenoy, R. Guglani, J. S. Sandhu. Effectiveness of an aerobic walking program using heart rate monitor and pedometer on the parameters of diabetes control in Asian Indians with type 2 diabetes. Primary Care Diabetes, vol. 4 , vol. 1, pp. 41-45, 2010.

[8] C. M. Perez-Terzic. Exercise in cardiovascular diseases. Physical Medicine and Rehabilitation, vol.4, no. 11, pp. 867-873, 2012. 
[9] J. She, H. Nakamura, K. Makino, Y. Ohyama, H. Hashimoto, M. Wu. Experimental selection and verification of maximum-heart-rate formulas for use with Karvonen formula. In Proceedings of the 10th International Conference on Informatics in Control, Automation and Robotics, SCITEPRESS, Reykjavik, Iceland, pp. 536-541, 2013.

[10] K. Onodera, M. Miyashita. A study on Japanese scale for rating of perceived exertion in endurance exercise. Japan Journal of Physical Education, Health and Sport Sciences, vol. 21, no. 4, pp. 191-203, 1976. (in Japanese)

[11] G. Borg. Borg's Perceived Exertion and Pain Scales, Champagne: Human Kinetics, 1998.

[12] P. O. Ȧstrand, K. Rodahl, H. A. Dahl, S. B. Stromme. Textbook of Work Physiology: Physiological Bases of Exercise, 4th ed., Champagne: Human Kinetics, 2003.

[13] Sports Incubation System. Science of Sports, Tokyo: Natsumesha Co. Ltd., 2002. (in Japanese)

[14] ACSM: American College of Sports Medicine Position Stand, [online], Available: http://www.acsm.org/accesspublic-information / position-stands.

[15] H. Suzuki. Creating healthful physical exercise across generations. Kanto Gakuin University Society of Humanity and Environment Bulletin, vol. 8, pp. 1-16, 2007. (in Japanese)

[16] O. Inbar, A. Oten, M. Scheinowitz, A. Rotstein, R. Dlin, R. Casaburi. Normal cardiopulmonary responses during incremental exercise in 20-70-yr-old men. Medicine and Science in Sports and Exercise, vol. 26, no. 5, pp. 538-546, 1994.

[17] W. C. Miller, J. P. Wallace, K. E. Eggert. Predicting max $\mathrm{HR}$ and the HR-VO2 relationship for exercise prescription in obesity. Medicine and Science in Sports and Exercise, vol. 25, no. 9, pp. 1077-1081, 1993.

[18] H. Tanaka, K. D. Monahan, D. R. Seals. Age-predicted maximal heart rate revisited. Journal of the American College of Cardiology, vol. 37, no. 1, pp. 153-156, 2001.

[19] M. Gulati, L. J. Shaw, R. A. Thisted, H. R. Black, C. N. B. Merz, M. F. Arnsdorf. Heart rate response to exercise stress testing in asymptomatic women. Circulation, vol. 122, no. 2, pp. 130-137, 2010.

[20] B. R. Londeree, M. L. Moeschberger. Effect of age and other factors on maximal heart rate. Research Quarterly for Exercise and Sport, vol. 53, no. 4, pp. 297-304, 1982.

[21] Ministry of Education, Culture, Sports, Science and Technology: Statistical Abstract 2012 Ed. 3 Physical Education and Sports, [on-line], Available: http://www.mext.go.jp/english/statistics/1302984.htm.

[22] A. H. Shaldehi. Using Eta $(\eta)$ correlation ratio in analyzing strongly nonlinear relationship between two Variables in Practical researches. Journal of Mathematics and Computer Science, vol. 7, no. 3, pp. 213-220, 2013.

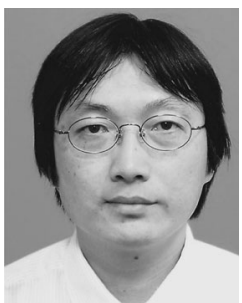

Jinhua She received his B. Sc. degree in engineering from Central South University, China in 1983, and his M.Sc. degree in 1990 and his Ph. D. degree in engineering from the Tokyo Institute of Technology, Japan in 1993. In 1993, he joined the Department of Mechatronics, School of Engineering, in 1993 University of Technology; and in April, 2008, he transferred to the university's School of Computer Science, where he is currently a professor. He received the Control Engineering Practice Paper Prize of the International Federation of Automatic Control (IFAC) in 1999 (jointly with M. Wu and M. Nakano).
His research interests include the application of control theory, repetitive control, process control, Internet-based engineering education, and robotics.

E-mail: she@stf.teu.jp.cn (Corresponding author)

ORCID iD: 0000-0003-3165-5045

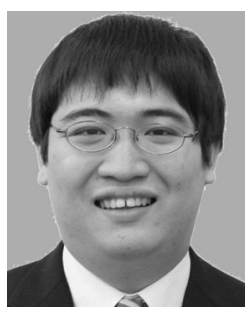

Hitoshi Nakamura received his B. Sc. degree from the Tokyo University of Technology, Japan in 2001. Currently, he is working on his masters degree at the university's Graduate School of Bionics, Computer and Media Sciences.

His current research interests include methods of evaluating degree of fatigue and the application of system theory in rehabilitation

E-mail: wotti2000@yahoo.co.jp

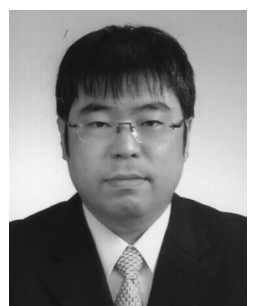

Koji Makino received his B.Sc., M. Sc. and $\mathrm{Ph}$. D. degrees in engineering from the Tokyo Institute of Technology, Japan in 1999, 2001, and 2008, respectively. In 2008, he joined the Research Organization for Information Science \& Technology. In June 2009, he joined the School of Computer Science, Tokyo University of Technology. And in April 2013, he joined the Interdisciplinary Graduate School of Medicine and Engineering, University of Yamanashi, where he is currently an assistant professor.

His research interests include the application of control theory, swarm robotics, and haptic devices.

E-mail: kohjim@yamanashi.ac.jp

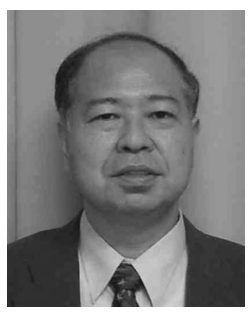

Yasuhiro Ohyama received his B. Sc., M. Sc. and Ph. D. degrees in engineering from the Tokyo Institute of Technology, Japan in 1980, 1982, and 1985, respectively. From 1985 to 1991, he worked on developing controllers for industrial robots and on CAD systems for control design as the director of the Advanced Control Laboratory Inc., Japan. He is currently a professor in the School of Computer Science, Tokyo University of Technology, Japan, where he does research on the application of control theory, robotics, and engineering education. He is a member of the Society of Instrument and Control Engineers (SICE) and the Institute of Electrical Engineers of Japan (IEEJ).

His research interests include the application of control theory, robotics, and engineering education.

E-mail: ohyama@stf.teu.ac.jp

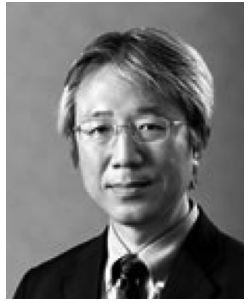

Hiroshi Hashimoto received his $\mathrm{Ph}$. D. degree in science and engineering from Waseda University, Japan in 1990 . He is currently a professor in the Master Program of Innovation for Design and Engineering, Advanced Institute of Industrial Technology, where he does research on intelligent robots, cybernetic interfaces, vision systems, welfare technology, and elearning. He is a member of the IEEE, the Society of Instrument and Control Engineers (SICE), and the Institute of Electrical Engineers of Japan (IEEJ).

His current research interests include mechatronics and the application of control theory.

E-mail: hashimoto@aiit.ac.jp 